\title{
Selection criteria for engineering solutions to ensure acoustic comfort of urban and rural areas
}

\author{
Vadim Bespalov ${ }^{1}$, Oksana Gurova ${ }^{1}$, Natalya Samarskaya ${ }^{1, *}$, and Valeriya Baklakova ${ }^{2,}$ \\ ${ }^{1}$ Don State Technical University, Gagarina sq. 1, 344022, Rostov-on-Don, Russia \\ ${ }^{2}$ Institute of Service and Business, Shevchenko st.147, 346500, Shakhty, Russia
}

\begin{abstract}
The article reflects the opinion that the choice of optimal engineering solution to ensure acoustic comfort in a specific urban or rural area should be based, first of all, on the use of two criteria: environmental efficiency and energy efficiency. The article proposes using, along with environmental efficiency, an additional optimization criterion - energy efficiency of the process for modern conditions of multi-criteria selection of engineering solutions in the field of providing acoustic comfort (ecological safety) in urban and rural areas. The article gives a justification for the need to apply the criterion of energy efficiency in this area and also presents the results of the obtained mathematical dependencies.
\end{abstract}

\section{Introduction}

Despite the annual increase in environmental safety requirements for urban space, megacities still cannot be called a favorable living environment[1-4]. Along with positive urban development trends in organizing a favorable aesthetic environment, transport accessibility, and functional zoning of urban areas, environmental problems such as pollution of chemical and energy origin are even aggravated[5-6]. Together, these problems cause various kinds of diseases, both in the adult and in the children's population. Moreover, of all types of diseases, in the adult population, diseases of the central nervous system ( $40 \%$ of all diseases), respiratory diseases $(30 \%)$ and circulatory system diseases $(12 \%)$ predominate. Analyzing the causes of diseases and the effects of adverse environmental factors in the urban environment, we can conclude that acoustic discomfort makes a significant contribution to the development of many diseases[7-8]. Auditory adaptation to increased levels of noise in a city dweller does not protect him from pathological processes, the result of which are disorders in the central nervous system, which manifest as nervous exhaustion, mental depression, autonomic neurosis. At the same time, the elderly, who are predominant in number, are most sensitive to the effect of increased noise levels[9-10]. Therefore, the issues of ensuring the environmental safety of urban space should be considered, first of all, from the position of creating acoustic comfort by choosing rational noise protection measures.

*Corresponding author: nat-samars@yandex.ru 


\section{Theory or experimental methods}

The rational choice of various engineering solutions to reduce noise in urban areas should be, above all, scientifically reasoned. Any scientific justification is based on a specific theory or scientific approach, containing appropriate methods, calculated dependencies, criteria for the implementation of the process as a whole or its individual stages.

In the framework of the tasks we are solving related to ensuring acoustic comfort in urban areas by choosing engineering solutions, the closest, in our opinion, is the physical and energy concept described in [11-12]. It is this concept that allows to evaluate and compare various technologies based on two criteria:

1) environmental efficiency of the process,

2) energy efficiency of the process.

The essence of the chosen concept is to consider physical characteristics of two interrelated processes: acoustic pollution of the urban environment and its reduction. Each of the processes includes several stages (Figure 1).

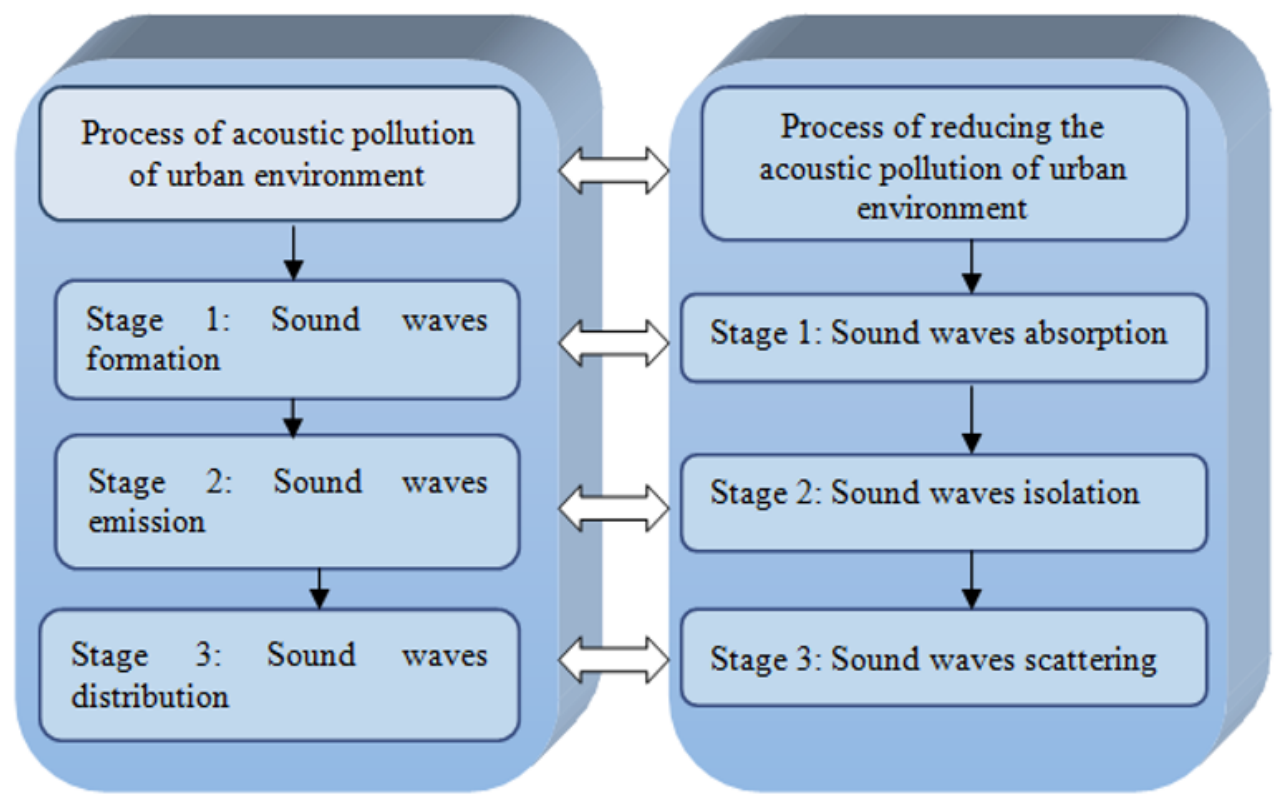

Fig.1 Structure of the relationship of the processes of urban environmentacoustic pollution and its reduction.

The main physical object at all stages of two interconnected processes are sound waves that change the values of their parameters (vibration frequency, propagation speed, sound intensity) at each stage. By deliberately exerting an external suppressive effect on sound waves at each stage, it is possible to achieve a reduction in the acoustic pollution of the urban environment.

When choosing the functional elements of a noise control system, there is a need to use evaluation criteria, the main of which we called the environmental efficiency and energy efficiency of the process of acoustic pollutionreduction.

The application of these two criteria is due to the existing problem of the choice of various measures, methods, ways and technical means. Currently, there are many modern engineering solutions that allow solving environmental safety problems, including ensuring acoustic comfort in urban areas [13-16]. Traditionally, the resulting process efficiency 
parameter is identified with the concept of efficiency which contradicts the classical definition of efficiency as an energy process evaluation. We considered the environmental efficiency of the process of reducing urban noise through the effectiveness of individual stages of this process: sound absorption, sound insulation and sound scattering, considered in [11]. At the same time, we obtained a mathematical description of the physical nature of the process of reducing acoustic pollution in urban areas (RAP):

$$
P_{R A P}=1-\left(1-P_{R P E}\right)\left(1-P_{R P A}\right)=1-\left[1-\left(1-P_{A S}\right)\left(1-P_{I S}\right)\right]\left[1-\left(1-P_{S S}\right)\right]
$$

Formula (1) characterizes the probability of implementation of the process of reducing the acoustic pollution of an urban area $\left(P_{R A P}\right)$, as the set of probabilities of the physical processes of decreasing the acoustic pollution of technological equipment $\left(P_{R P E}\right)$, including the absorption $\left(P_{A S}\right)$ of sound waves and sound insulation $\left(P_{I S}\right)$, as well as the reduction of air acoustic pollution $\left(P_{R P A}\right)$, including scattering of sound waves $\left(P_{S S}\right)$.

\section{Results and Discussion section}

Based on the probabilistic description of the acoustic pollution reduction process, it can be concluded that the implementation efficiency of each stage of the process is adequate to the probability of its implementation:

$$
P_{i} \sim E_{e f},
$$

Then, taking into account the mathematical description of the physical nature of the acoustic pollution reduction process of an urban area for environmental efficiency, the following dependency could be compiled:

$$
E_{e f R A P}=1-\left[1-\left(1-E_{e f A S}\right)\left(1-E_{\text {efIS }}\right)\right]\left[1-\left(1-E_{\text {efSS }}\right)\right],
$$

where Eef ${ }_{A S}$, Eef ${ }_{I S}$, Eef ss, - are accordingly, the effectiveness of the implementation of the processes of sound absorption, sound insulation and sound scattering.

It should be emphasized that the analysis of publications by world scientists [17-18] showed that the concept of process efficiency was determined by the authors only for individual processes, for example, sound absorption. In this case, by efficiency is meant the difference in sound pressure levels before and after sound absorption, respectively, related to the initial sound pressure level. And the required effectiveness is determined by the formula:

$$
E_{e f(r e g)}=\frac{\left(L_{i n}-P e r L e v\right)}{L_{i n}},
$$

where: Lin is initial measured equivalent sound pressure level dBA; Per Lev is the maximum permissible equivalent level of acoustic exposure, adopted in accordance with the regulatory document, $\mathrm{dBA}$.

This definition can be considered acceptable for assessing the remaining stages of the process of reducing acoustic pollution. It is only necessary to clarify the concepts of the initial and final sound pressure levels at specific spatial points, based on the purpose of each stage of the process of reducing acoustic pollution. The authors constructed a physical model for reducing the acoustic pollution of the urban environment, where each stage of this process can be analyzed separately (Figure 2) 


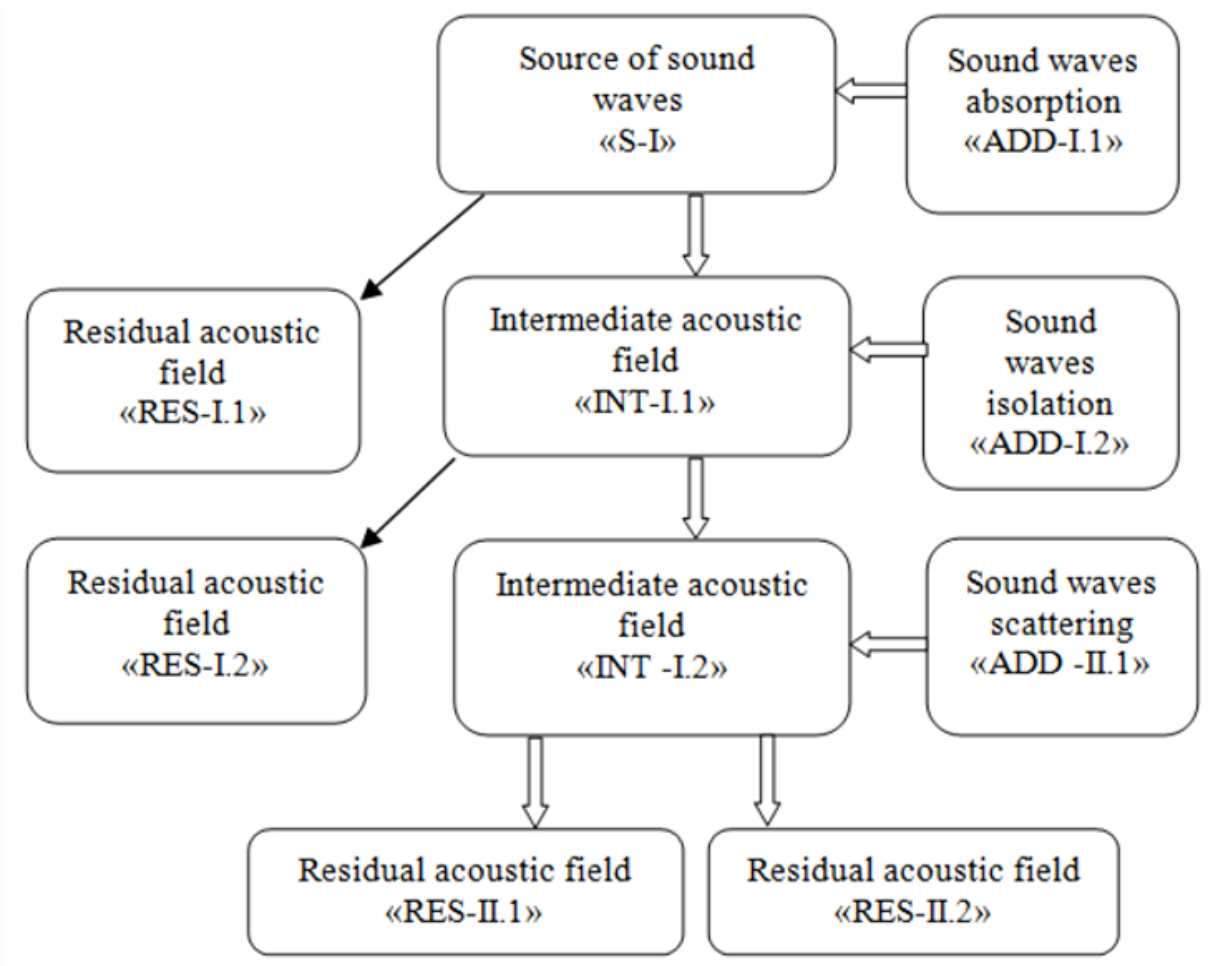

Fig. 2 A physical model for reducing acoustic pollution in an urban environment.

So, when organizing the process of absorption of the generated sound waves, the vibrational energy of the sound wave goes into heat (dissipates) due to friction losses in the sound absorber. Sound absorbers are porous materials, i.e. having an inconsistent structure, since the friction losses in them are most significant. In the process of absorption of sound waves, the acoustic field interacts with a specially created additional acoustic field with the goal of its maximum destruction, characterized by the sound absorption efficiency Eef AS. As a result of this interaction, two acoustic fields are formed:

- INT-I.1 an intermediate acoustic field, which is sound waves with a certain reduced level of sound pressure, directed either directly to the environment (if Per Lev is observed), or to the soundproofing zone;

- RES-I.I residual acoustic field, which is sound waves with a certain reduced level of sound pressure remaining in the surrounding space.

When organizing sound insulation in the process of emitting sound waves, sound power decreases along the path of propagation of sound waves, this is achieved using soundproof fencing, soundproofing booths and control panels, soundproofing covers and acoustic screens.

In the process of soundproofing INT-I.1, an acoustic field interacts with a specially created $A D D-I .2$ acoustic field with the aim of its maximum destruction. As a result of this interaction, two acoustic fields are formed:

- INT-I.2 intermediateacoustic field, which is sound waves with a certain reduced level of sound pressure, directed either directly to the environment (if Per Lev is observed), or to the zone of forced scattering of sound waves;

- RES-I.2 residual acoustic field, which is sound waves with a certain reduced level of sound pressure remaining in the surrounding space. 
In the process of scattering of sound waves along the path of their propagation, they ensure the achievement of standard values of noise parameters in an environmentally significant zone. The basis of this method is a change in the directivity of the emission of sound waves, but this is possible in case when sound waves are emitted in a directional manner. The directional sound wave should be oriented in the opposite direction from the

In the process of forced scattering of sound waves INT-I.2, the acoustic field interacts with a previously prepared external $A D D-I I .1$ additional acoustic field. As a result of this interaction, two acoustic fields are formed:

- RES-II.1 residual acoustic field, which is represented by the minimum number of sound waves, the sound pressure level of which must correspond to Per Lev;

- RES-II.2 residual acoustic field, which is represented by the maximum number of sound waves passing into the surface layer of the atmosphere.

Development of modern means and methods of reducing the noise of the urban environment today allows us to propose several different engineering solutions to achieve the same value of the required efficiency, which indicates the inadequacy of using only efficiency as an optimization criterion.

Therefore, at present, the choice of engineering solutions is a multicriteria task and involves the use in addition to the main criterion (efficiency) of additional criteria, such as energy-intensive indicator; indicator of technological reliability; indicator of economic efficiency.

In our chosen concept, under the energy efficiency of $E e f^{f n}(\%)$ we understand the ratio of specific energy $W(\mathrm{~W})$ spent on achieving the goals of the noise reduction process as a whole and its individual stages to specific energy $N(\mathrm{~W})$ spent on creating directed external influences on the acoustic field (i.e. "additional" acoustic fields).

To assess the effectiveness of the use of energy activity from the point of view of reflection and absorption of sound energy for noise protection means, we obtained a mathematical dependence:

$$
E_{e f}^{e n}=\frac{\left(\frac{\alpha^{2}(1-\alpha) c_{S w} h b}{\exp \left(\frac{\ln (1-\alpha) h b f n}{c_{S w} \rho} r\right)}+1\right) E_{e f}}{c_{S w} \alpha^{2} h b} 100
$$

where: $c_{s w}$ is sound wave propagation speed, $\mathrm{m} / \mathrm{s} ; p$ is density of the medium, $\mathrm{kg} / \mathrm{m} ; \eta$ is coefficient of viscosity of the medium, $\mathrm{P}$; $h$ is thickness of the material of the noise protection means, in the direction normal to the direction of movement of the sound wave, $\mathrm{m}$.

The criterion of energy efficiency of noise reduction also allows to evaluate the use of a complex of several methods (devices) used in one noise reduction system.

\section{Conclusion}

Thus, the search for optimal engineering solutions to ensure the acoustic comfort of urban areas should be based, first of all, on the criteria of environmental efficiency and energy efficiency. It is these criteria that make it possible to rationally make the choice of soundproofing technologies both for the entire process of acoustic pollution in general and for its individual stages: sound absorption, sound insulation and sound scattering.

Such a scientific result can find practical application in the design of various technologies for reducing city noise, in the comprehensive territorial planning of cities, as well as in the development of environmental protection programs and ensuring the environmental safety of populated areas. 


\section{References}

1. M. Mapar, Ecological Indicators, 83, 271-191 (2017) doi.org/10.1016/j.ecolind.2017.08.012

2. Y. Sylvia, J. He, J Thøgersen, Research in Transportation Economics, 62, 57-67,(2017) doi.org/10.1016/j.retrec.2017.03.004

3. Q. Zhong, J. Ma, B. Zhao, X. Wang, X. Xiao, Remote Sensing of Environment, 233, (2019) doi.org/10.1016/j.rse.2019.111374

4. E. Kulińska, M. Dendera - Gruszka, Transportation Research Procedia, 39, 242251(2019)

5. G. Liu, Y. Zhifeng, B. D. Fath, L. Shi, S. Ulgiati, Applied Energy, 186(215), 96-114 (2017) doi.org/10.1016/j.apenergy.2016.06.132

6. M. Yuan, Ch. Yin, Y. Sun, W. Chen, Sustainable Cities and Society, 50, (2019) doi.org/10.1016/j.scs.2019.101678

7. S. Sohrabi, H. Khreis, Environment International, 136, (2020) doi.org/10.1016/j.envint.2020.105520

8. J. D.Thacher, A.H. Poulsen, O. Raaschou-Nielsen, Environmental Research, 182, (2020) doi.org/10.1016/j.envres.2019.109051

9. P. Arezes, G. Pereira, M. Kroger, P. Sampaio, Procedia Manufacturing, 3, 4565-4571 (2015)

10. Y. Yu, K. Paul, O. A. Arah, E. Rose Mayeda, B. Ritz, Environment International, 134, (2020)doi.org/10.1016/j.envint.2019.105269

11. V. I. Bespalov, N. S. Samarskaya, E. P. Lysova, V. V. Baklakova, N. V. Udina, AIP Conference Proceedings, 2188, 060010 (2019); https://doi.org/10.1063/1.5138479

12. V. Bespalov, N. Samarskaya, E. Lysova, O. Akay, Paper presented at the MATEC Web of Conferences, 170 (2018) doi:10.1051/matecconf/201817003010

13. L. Claudi, M. Arnesano, P. Chiariotti, G. Battista, G. M. Revel, Measurement, 146, 675-688 (2019)

14. Y. Jin, H. Jin, J. Kang, Building and Environment, 168, (2020) doi.org/10.1016/j.buildenv.2019.106517

15. V. Marusceac, M. Ciotlaus, Procedia Manufacturing, 32, 161-166 (2019)

16. J. Bohatkiewicz, Transportation Research Procedia, 14, 2744-2753 (2016)

17. K. Hirosawa, Applied Acoustics, 164, (2020) doi.org/10.1016/j.apacoust.2020.107222

18. L. Galbrun, L. Scerri, Building and Environment, 116, 130-139 (2017) 